\title{
Simulation studies for large scale acoustic neutrino detectors
}

\author{
Dominik Kießling ${ }^{1}$ and Robert Lahmann ${ }^{1, \star}$ \\ ${ }^{1}$ Friedrich-Alexander-Universität Erlangen-Nürnberg, Erlangen Centre for Astroparticle Physics, Erwin- \\ Rommel-Str. 1, 91058 Erlangen, Germany
}

\begin{abstract}
The AMADEUS system is a submarine acoustic array that was operated from 2008 until 2015 as a part of the ANTARES neutrino telescope in the Mediterranean Sea. Its design goal was to investigate the feasibility of acoustic neutrino detection in the deep sea. The data taken during its eight years of operation provide a wealth of information for setting up realistic simulations of future acoustic neutrino detectors. Using in addition simulations of neutrino interactions in water, various potential acoustic neutrino detector designs were investigated and methods for suppressing background and reconstructing energy and direction of incoming neutrinos were developed. In this article, some recent results will be presented.
\end{abstract}

\section{Introduction}

The acoustic test system AMADEUS [1] is an integral part of the ANTARES [2] neutrino telescope, located in the Mediterranean Sea. It comprises 36 acoustic sensors, which have been taking data from 2008 to 2015 in the context of a feasibility study towards acoustic neutrino detection in sea water. Acoustic particle detection is based on the thermoacoustic model which is described e.g. in [3-5] and references therein. It is a promising technique for the detection of neutrinos with energies near and above $10^{18} \mathrm{eV}$. The acoustic background recorded with AMADEUS was used in combination with simulated neutrino pulses to study the properties of potential future acoustic neutrino detector designs and to investigate neutrino detection and noise reduction techniques for such detectors. The next generation neutrino telescope $\mathrm{KM} 3 \mathrm{NeT}$ [6] will comprise an acoustic sensors as part of a position calibration system [7]. Simulations presented in the following are based on the design of a KM3NeT Building Block ${ }^{1}$, in which each optical module is equipped with an acoustic sensor.

\section{Classification of neutrinos}

Previous investigations of noise reduction and neutrino classification with the AMADEUS data have concentrated on methods that use signal features of individual acoustic sensors or local clusters of these sensors ${ }^{2}$ [8]. The neutrino classification presented here is described in [9] and extends the

\footnotetext{
^e-mail: robert.lahmann@fau.de

${ }^{1} \mathrm{~A}$ KM3NeT building block is a three-dimensional arrangement of 2070 optical modules for the detection of Cherenkov light emitted from charged particles resulting from neutrino interactions.

${ }^{2}$ Six acoustic sensors, mechanically combined to a so-called storey with fixed inter-sensor spacings of roughly $1 \mathrm{~m}$, form a natural cluster within the AMADEUS device.
} 


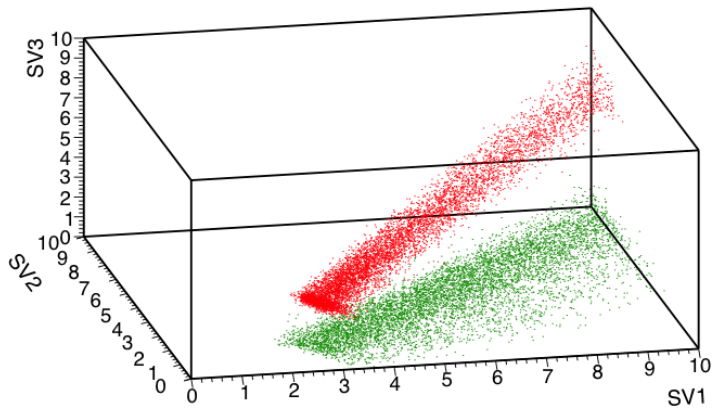

Figure 1. Decomposition in singular values SV1, SV2, and SV3 of the hit distribution in the detector for neutrinos (green, band in SV1-SV2 plane) and background (red, band with pronounced SV3 component) as described in the text. The neutrino signals can be effectively discriminated from the background with a cut on the SV3 parameter (spread of data points in direction perpendicular to the fitted plane).

analysis to the events remaining after all the aforementioned data selection steps on sensor and cluster level have been carried out. A vector of 16 features was derived, allowing for the identification of the specific disk-shaped propagation pattern of the acoustic neutrino signal in a three-dimensional volume of water, the so-called pancake. The feature vector is used as input to machine learning algorithms that perform the classification.

For both the training of the algorithms and the analysis, neutrino interactions were simulated with energies in the range $10^{18}-10^{21} \mathrm{eV}$. As background, signals of the positioning system, spherically emitting sources (as e.g. from dolphins and marine traffic) and random coincidences were considered. As an example of one of the 16 features, the result of a singular value decomposition of the hit distribution in the detector is shown in Fig. (1). It essentially corresponds to a least-square fit of a plane to the distribution, where the three singular values are measures of the spread of the hits in two orthogonal directions in the plane and one direction perpendicular to the plane. The acoustic "pancake" is hence reconstructed as a by-product, yielding the direction of the incoming neutrino and the vertex position of its interaction.

Using the classification based on the aforementioned feature vector (after storey-based background rejection), $99.1 \%$ of neutrinos are recognized correctly while $0.0007 \%$ of background is misidentified as signal events. For the background rates obtained with AMADEUS, this corresponds to $O(1)$ background event per year being misclassified as neutrino.

\section{Reconstruction of neutrino energy and direction}

For the feature vector discussed in the previous section, the parameters of the event were reconstructed independently from each other. One of these parameters is the shower energy, which is estimated from the signal amplitudes measured in individual sensors, the result having a strong dependence on the precision of the reconstruction of the neutrino direction and interaction vertex. The uncertainty of the energy reconstruction can be strongly reduced by allowing for a simultaneous optimisation of all parameters in the reconstruction, as presented in [9]. Such a combined fit yields more accurate results for all involved values, i.e. besides the energy also for the neutrino direction and interaction vertex position.

Results of this combined fit and a comparison with the estimate obtained from the "energy only" fit used for the feature vector described in the previous section are shown in Fig. (2). For the reconstruction using the combined fit, the error on the reconstructed energy is less then $75 \%$ of the true energy for $90 \%$ of 20000 reconstructed neutrinos. The angular resolution was found to be about $1^{\circ}, 3$ with an average vertex error of about $250 \mathrm{~m}$.

\footnotetext{
${ }^{3}$ This assumes all detected neutrinos are originating from the upper hemisphere. As substantiated in [9], a direction ambiguity arises near the horizon.
} 


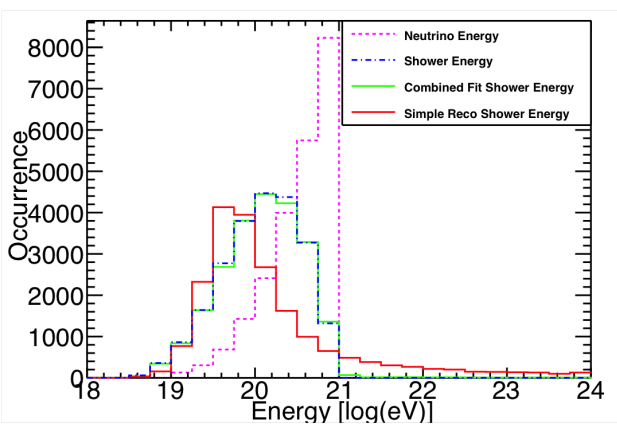

Figure 2. A comparison of the two different energy reconstruction methods. The dotted (dashed) histogram shows the true energy of the neutrino (shower). The shower energy reconstructed with the combined fit is displayed as a solid green (light) line, while the simple method from the feature extraction is shown in red (dark). The entries in this plot were generated for a KM3NeT Building Block. The bin size is larger than the typical error on the energy obtained from the combined fit. See [9] for further details.

\section{Summary and conclusions}

Advanced techniques for the reconstruction of acoustic signals and improved methods for the identification of neutrinos were presented. They were developed using background measured with the AMADEUS acoustic system for simulations of potential future acoustic submarine arrays. The neutrino identification exploits the characteristic three-dimensional shape of the acoustic pressure field to significantly reduce the expected background rate. A combined fit of shower energy, interaction vertex and direction of the incoming neutrino yields a substantial improvement of the precision of the reconstruction of these parameter over reconstructing them individually. Albeit not designed for acoustic detection, the next generation neutrino telescope KM3NeT will contain acoustic sensors for the purpose of position calibration, which may be used in parallel for further investigations of acoustic neutrino detection techniques.

Given the expected scaling of the flux of neutrinos with energy in the vicinity of $10^{18} \mathrm{eV}$, reducing the trigger threshold on the level of individual acoustic sensors seems crucial. Investigations carried out in [9] indicate that deriving methods to reduce the threshold on the amplitude of triggered signals without increasing the number of triggered random coincidences to an unsustainable level is a crucial task to be pursued for future acoustic neutrino detectors.

\section{Acknowledgements}

The AMADEUS project was supported by the German government (Bundesministerium für Bildung und Forschung, BMBF) through grants 05A08WE1 and 05A11WE1.

\section{References}

[1] J.A. Aguilar et al. (ANTARES Coll.), Nucl. Inst. and Meth. A 626-627, 128 (2011)

[2] M. Ageron et al. (ANTARES Coll.), Nucl. Inst. and Meth. A 656, 11 (2011)

[3] R. Lahmann et al., Astropart. Phys. 65, 69 (2015)

[4] J.G. Learned, Phys. Rev. D 19, 3293 (1979)

[5] G.A. Askariyan et al., Nucl. Inst. and Meth. 164, 267 (1979)

[6] S Adrián-Martínez et al. (KM3NeT Coll.), J. Phys. G: Nucl. Part. Phys. 43, 084001 (2016)

[7] S. Viola et al., KM3NeT acoustic positioning and detection system, in Proceedings of ARENA 2018, Catania (Italy), June 12-15 (2018), to be published in: EPJ Web of Conf.

[8] M. Neff, Ph.D. thesis, Univ. Erlangen-Nürnberg (2013), ECAP-2013-023

[9] D. Kießling, Ph.D. thesis, Univ. Erlangen-Nürnberg (2018), to be published 